\title{
Molecular investigation of mutation in PncA gene for pyrazinamide resistance in Mycobacterium tuberculosis: an in silico approach
}

\author{
D. Ruban Durairaj*, P. Shanmughavel \\ Department of Bioinformatics, Bharathiar University, Coimbatore, India \\ *e-mail: ruban.bioinfo@gmail.com
}

Key words: pyrazinamide, PncA gene, mutations, Mycobacterium tuberculosis, in silico

Motivation and Aim: PncA is a gene coding for the enzyme pyrazinamidase in Mycobacterium [1].The pyrazinamidase enzyme converts the pyrazinamide into its active form pyrazinoic acid [2]. There is significant relation between the mutations in PncA gene and the pyrazinamide resistance of $M$. tuberculosis. There are several mutations in PncA gene identified and reported all over the world from the year 1996 to till date [2]. The main aim of this study is to investigate those mutations at molecular level by applying in silico approaches.

Methods and Algorithms: We modelled the structure of the enzyme pyrazinamidase encoded by PncA gene, with I-Tasser tool. The modelled structure is validated by using Ramachandran plot. Then the modelled structure is subjected to molecular docking with the pyrazinamide drug by utilizing Schrodinger Maestro software. Then the apo structure and docked complex were subjected to molecular dynamics simulation by using GROMACS software package.

Results: The mutation at the nucleotide position 202, mutates the residue $\mathrm{W}$ in wild protein to $\mathrm{R}$ in mutant which is disfavoured substitution. This may leads to the pyrazinamide resistance. In molecular docking study we identified that the pyrazinamide drug is binding well with the wild protein whereas it lacks its binding activity in the mutant protein. The binding scores are -4.34 in wild protein and -3.21 in mutant protein. The stability of the complexes were further checked with molecular dynamics simulation studies.

Conclusion: The results obtained in this study suggesting that the mutation at the nucleotide position 202 leads to the pyrazinamide resistance in Mycobacterium tuberculosis. Further experimental investigation at that mutation will give us fruitful results in future. Hence we are suggesting that the PncA mutant protein is resistance to pyrazinamide drug, we can consider it as active drug target for finding the drug alternate of pyrazinamide to treat tuberculosis.

Acknowledgements: We acknowledge DBT-Centre for Bioinformatics, Bharathiar University, Coimbatore, Tamilnadu, India for providing all the computational facilities to carry out this work.

\section{References}

1. Whitfield M.G., Soeters H.M., Warren R.M., York T., Sampson S.L., Streicher E.M., van Helden P.D., van Rie A. (2015) A global perspective on pyrazinamide resistance: systematic review and meta-analysis. PLoS One. 10(7):e0133869. DOI 10.1371/journal.pone.0133869.

2. Juréen P., Werngren J., Toro J.-C., Hoffner S. (2016) Pyrazinamide resistance and pncA gene mutations in Mycobacterium tuberculosis. Antimicrob. Agents Chemother. 52(5):1852-1854. DOI 10.1128/ AAC.00110-08. 